\section{Crystal structure of $(E)-N^{\prime}-\{[(1 R, 3 R)-3-$ isopropyl-1-methyl-2-oxocyclopentyl]- methylidene\}-4-methylbenzenesulfono- hydrazide}

\author{
David Tymann, Dina Christina Dragon, Christopher Golz, \\ Hans Preut,* Carsten Strohmann and Martin Hiersemann \\ Fakultät für Chemie und Chemische Biologie, Technische Universität Dortmund, \\ Otto-Hahn-Strasse 6, 44221 Dortmund, Germany. *Correspondence e-mail: \\ hans.preut@tu-dortmund.de
}

Received 25 November 2014; accepted 5 December 2014

Edited by E. R. T. Tiekink, University of Malaya, Malaysia

The title compound, $\mathrm{C}_{17} \mathrm{H}_{24} \mathrm{~N}_{2} \mathrm{O}_{3} \mathrm{~S}$, was synthesized in order to determine the relative configuration of the corresponding $\beta$-keto aldehyde. In the U-shaped molecule, the fivemembered ring approximates an envelope with the methylene atom adjacent to the quaternary $\mathrm{C}$ atom being the flap. The dihedral angles between the four nearly coplanar atoms of the five-membered ring and the flap and the aromatic ring are 38.8 (4) and $22.9(2)^{\circ}$, respectively. The bond angles around the $\mathrm{S}$ atom are in the range $104.11(16)-119.95(16)^{\circ}$. In the crystal, molecules are linked via $\mathrm{N}-\mathrm{H} \cdots \mathrm{O}$ by hydrogen bonds, forming a chain along the $a$-axis direction.

Keywords: crystal structure; benzenesulfonohydrazide; terpenoid-related building blocks; hydrogen bonding; cyclopentanoids.

CCDC reference: 1437665

\section{Related literature}

For the synthesis of terpenoid-related building blocks, in particular cyclopentanoids, see: Becker et al. (2013); Gille et al. (2011); Helmboldt et al. (2006); Nelson et al. (2011); Tymann et al.(2014). For a review on cyclopentanoids by ring contraction see: Silva (2002). For a solid-acid catalysed rearrangement of cyclic $\alpha, \beta$-epoxy ketones see: Elings et al. (2000).

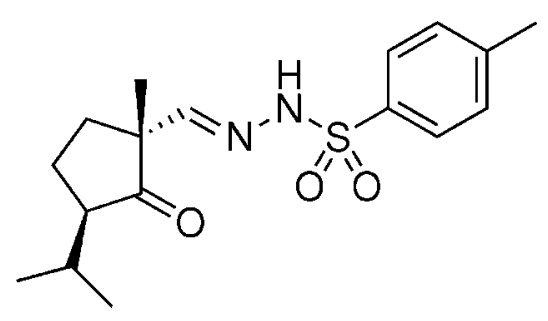

\section{Experimental}

\subsection{Crystal data}

$\mathrm{C}_{17} \mathrm{H}_{24} \mathrm{~N}_{2} \mathrm{O}_{3} \mathrm{~S}$

$M_{r}=336.44$

Monoclinic, $P 2_{1}$

$a=6.6198(8) \AA$

$b=16.8318(18) \AA$

$c=7.9506(9) \AA$

$\beta=97.141(11)^{\circ}$

$$
\begin{aligned}
& V=879.00(17) \AA^{3} \\
& Z=2 \\
& \text { Mo } K \alpha \text { radiation } \\
& \mu=0.20 \mathrm{~mm}^{-1} \\
& T=173 \mathrm{~K} \\
& 0.23 \times 0.10 \times 0.03 \mathrm{~mm}
\end{aligned}
$$

\subsection{Data collection}

Oxford Diffraction Xcalibur Sapphire3 diffractometer

Absorption correction: multi-scan (CrysAlis CCD; Oxford Diffraction, 2008)

$T_{\min }=0.98, T_{\max }=1.00$

\subsection{Refinement}

$R\left[F^{2}>2 \sigma\left(F^{2}\right)\right]=0.044$

$w R\left(F^{2}\right)=0.091$

$S=1.01$

3684 reflections

216 parameters

1 restraint

$\mathrm{H}$ atoms treated by a mixture of independent and constrained refinement

6620 measured reflections 3684 independent reflections 3185 reflections with $I>2 \sigma(I)$ $R_{\text {int }}=0.032$

Table 1

Hydrogen-bond geometry $\left(\AA,^{\circ}\right)$.

\begin{tabular}{lllll}
\hline$D-\mathrm{H} \cdots A$ & $D-\mathrm{H}$ & $\mathrm{H} \cdots A$ & $D \cdots A$ & $D-\mathrm{H} \cdots A$ \\
\hline $\mathrm{N} 1-\mathrm{H} 1 N \cdots \mathrm{O}^{\mathrm{i}}$ & $0.91(4)$ & $2.03(4)$ & $2.889(4)$ & $158(3)$ \\
\hline
\end{tabular}

Symmetry code: (i) $x-1, y, z$.

Data collection: CrysAlis CCD (Oxford Diffraction, 2008); cell refinement: CrysAlis CCD (Oxford Diffraction, 2008); data reduction: CrysAlis RED (Oxford Diffraction, 2008); program(s) used to solve structure: SHELXS97 (Sheldrick, 2008); program(s) used to refine structure: SHELXL2013 (Sheldrick, 2008); molecular graphics: SHELXTL-Plus (Sheldrick, 2008); software used to prepare material for publication: SHELXL97 (Sheldrick, 2008) and PLATON (Spek, 2009).

\title{
Acknowledgements
}

Financial support obtained from the Beilstein Institut is gratefully acknowledged 


\section{data reports}

Supporting information for this paper is available from the $\mathrm{IUCr}$ electronic archives (Reference: TK5349).

\section{References}

Becker, J., Butt, L., von Kiedrowski, V., Mischler, E., Quentin, F. \& Hiersemann, M. (2013). Org. Lett. 15, 5982-5985.

Elings, J. A., Lempers, H. E. B. \& Sheldon, R. A. (2000). Eur. J. Org. Chem. pp. 1905-1911.

Gille, A., Rehbein, J. \& Hiersemann, M. (2011). Org. Lett. 13, 2122-2125.
Helmboldt, H., Köhler, D. \& Hiersemann, M. (2006). Org. Lett. 8, 1573-1576. Nelson, B., Hiller, W., Pollex, A. \& Hiersemann, M. (2011). Org. Lett. 13, 4438 4441.

Oxford Diffraction (2008). CrysAlis CCD and CrysAlis RED. Oxford Diffraction Ltd, Yarnton, England.

Parsons, S. \& Flack, H. (2004). Acta Cryst. A60, s61.

Sheldrick, G. M. (2008). Acta Cryst. A64, 112-122.

Silva, L. F. Jr (2002). Tetrahedron, 58, 9137-9161.

Spek, A. L. (2009). Acta Cryst. D65, 148-155.

Tymann, D., Klüppel, A., Hiller, W. \& Hiersemann, M. (2014). Org. Lett. 16, 4062-4065. 


\section{supporting information}

Acta Cryst. (2015). E71, o99-o100 [doi:10.1107/S2056989014026747]

\section{Crystal structure of $(E)-N^{\prime}-\{[(1 R, 3 R)$-3-isopropyl-1-methyl-2-oxocyclopentyl]- methylidene\}-4-methylbenzenesulfonohydrazide}

\section{David Tymann, Dina Christina Dragon, Christopher Golz, Hans Preut, Carsten Strohmann and Martin Hiersemann}

\section{S1. Comment}

Prompted by our efforts in natural product synthesis, we seek access to cyclopentyl units. Herein, we chosed a ring contraction strategy of a cyclic epoxy ketone. A Brønsted-acid promoted [1,2]-sigmatropic rearrangement of cispiperitone oxide delivered trans-3-Isopropyl-1-methyl-2-oxocyclopentane-1-carbaldehyde (II). A subsequent condensation of (II) with $p$-toulenesulfonyl hydrazide afforded the title compound (I).

\section{S2. Experimental}

A sealable glass pressure tube was charged with a solution of trans-3-Isopropyl-1-methyl-2-oxocyclopentane-1carbaldehyde (II) $\left(\mathrm{C}_{10} \mathrm{H}_{16} \mathrm{O}_{2}, M=168.23 \mathrm{~g} / \mathrm{mol},[\alpha]_{\mathrm{D}}{ }^{20}=+248.3\right.$ (c $\left.\left.0.059 \mathrm{~mol} / L, \mathrm{CHCl}_{3}\right), 150 \mathrm{mg}, 0.89 \mathrm{mmol}, 1.0 \mathrm{eq}\right)$ and p-toluenesulfonyl hydrazide $\left(\mathrm{C}_{7} \mathrm{H}_{10} \mathrm{~N}_{2} \mathrm{O}_{2} \mathrm{~S}, M=186.23 \mathrm{~g} / \mathrm{mol}, 232 \mathrm{mg}, 1.24 \mathrm{mmol}, 1.4 \mathrm{eq}\right)$ in methanol (9 ml, 10.1 $\mathrm{ml} / \mathrm{mmol})$. The tube was sealed with a Teflon screw cap and placed in a pre-heated oil bath (353 K). After being stirred for $2.5 \mathrm{~h}$ at $353 \mathrm{~K}$, the reaction mixture was cooled to ambient temperature and stirred for additional $16 \mathrm{~h}$ at room temperature. Next, the volatiles were removed under reduced pressure. Purification of the residue by flash chromatography (cyclohexane/ethyl acetate $50 / 1$ to $5 / 1)$ delivered the title compound (I) $\left(\mathrm{C}_{17} \mathrm{H}_{24} \mathrm{~N}_{2} \mathrm{O}_{3} \mathrm{~S}, M=336.45\right.$ $\mathrm{g} / \mathrm{mol}, 161 \mathrm{mg}, 0.48 \mathrm{mmol}, 54 \%$ ) as a white solid and as an apparent mixture of double bond isomers (ratio = 68:32). Subsequent recrystallization of (I) from $n$-pentane provided colourless plates of the $E$-configured double bond isomer of (I). The ratio of isomers was determined by integration of the ${ }^{1} \mathrm{H}$ NMR signals at 6.66 p.p.m. (s, 1H) and 7.12 p.p.m. (s, $1 \mathrm{H}$ ). Characterization data are reported for the mixture of isomers. $R_{\mathrm{f}} 0.44$ (cyclohexane/ethyl acetate 2/1); m.p. $361-363$ $\mathrm{K} ;{ }^{1} \mathrm{H} \mathrm{NMR}\left(\mathrm{CDCl}_{3}, 500 \mathrm{MHz}\right.$, ratio of double bond isomers $\left.=68: 32\right) \delta 0.81\left(\mathrm{~d}, J=6.7 \mathrm{~Hz}, 3 \mathrm{H}^{\text {major }}\right), 0.93(\mathrm{~d}, J=6.9 \mathrm{~Hz}$, $\left.3 \mathrm{H}^{\text {minor }}\right), 0.97\left(\mathrm{~d}, J=6.7 \mathrm{~Hz}, 3 \mathrm{H}^{\text {major }}\right), 1.08-1.09\left(\mathrm{~m}, 3 \mathrm{H}^{\text {major }}+3 \mathrm{H}^{\text {minor }}\right), 1.12\left(\mathrm{~s}, 3 \mathrm{H}^{\text {minor }}\right), 1.52-1.59\left(\mathrm{~m}, 1 \mathrm{H}^{\text {minor }}\right), 1.62-1.81$ $\left(\mathrm{m}, 2 \mathrm{H}^{\text {major }}+3 \mathrm{H}^{\text {minor }}\right), 1.93-2.02\left(\mathrm{~m}, 1 \mathrm{H}^{\text {major }}\right), 2.05-2.23\left(\mathrm{~m}, 3 \mathrm{H}^{\text {major }}+1 \mathrm{H}^{\text {minor }}\right), 2.42\left(\mathrm{~s}, 3 \mathrm{H}^{\text {minor }}\right), 2.43\left(\mathrm{~s}, 3 \mathrm{H}^{\text {major }}\right), 2.53-2.56$ $\left(\mathrm{m}, 1 \mathrm{H}^{\text {minor }}\right), 6.66\left(\mathrm{~s}, 1 \mathrm{H}^{\text {minor }}\right), 7.12\left(\mathrm{~s} 1 \mathrm{H}^{\text {minor }}\right), 7.29-7.32\left(\mathrm{~m}, 2 \mathrm{H}^{\text {major }}+2 \mathrm{H}^{\text {minor }}\right), 7.58(\mathrm{br} . \mathrm{s}, 1 \mathrm{H}), 7.70(\mathrm{br} . \mathrm{s}, 1 \mathrm{H}), 7.79(\mathrm{~d}, J=$ $\left.8.2 \mathrm{~Hz}, 2 \mathrm{H}^{\text {major }}\right), 7.84\left(\mathrm{~d}, J=8.2 \mathrm{~Hz}, 2 \mathrm{H}^{\text {minor }}\right) ;{ }^{13} \mathrm{C} \mathrm{NMR}\left(\mathrm{CDCl}_{3}, 126 \mathrm{MHz}\right) \delta 16.9\left(\mathrm{CH}_{3}^{\text {minor }}\right), 18.5\left(\mathrm{CH}_{3}^{\text {major }}\right), 19.3$ $\left(\mathrm{CH}_{3}{ }^{\text {minor }}\right), 20.1\left(\mathrm{CH}_{3}{ }^{\text {major }}\right), 20.5\left(\mathrm{CH}_{2}^{\text {major }}\right), 20.9\left(\mathrm{CH}_{3}{ }^{\text {major }}\right), 21.57\left(\mathrm{CH}_{3}^{\text {minor }}\right), 21.59\left(\mathrm{CH}_{3}^{\text {major }}\right), 23.7\left(\mathrm{CH}_{3}^{\text {minor }}\right), 24.3\left(\mathrm{CH}_{2}{ }^{\text {minor }}\right)$, $26.2\left(\mathrm{CH}^{\text {major }}\right), 27.3\left(\mathrm{CH}^{\text {minor }}\right), 31.4\left(\mathrm{CH}_{2}^{\text {major }}\right), 35.8\left(\mathrm{CH}_{2}{ }^{\text {minor }}\right), 53.1\left(\mathrm{C}^{\text {major }}\right), 55.2\left(\mathrm{CH}^{\text {minor }}\right), 55.3\left(\mathrm{CH}^{\text {major }}\right), 61.9\left(\mathrm{C}^{\text {minor }}\right)$, $127.8\left(\mathrm{CH}^{\text {minor }}\right), 127.9\left(\mathrm{CH}^{\text {major }}\right), 129.4\left(\mathrm{CH}^{\text {minor }}\right), 129.5\left(\mathrm{CH}^{\text {major }}\right), 135.1\left(\mathrm{C}^{\text {major }}\right), 136.6\left(\mathrm{C}^{\text {minor }}\right), 134.8\left(\mathrm{C}^{\text {minor }}\right), 144.2\left(\mathrm{C}^{\text {major }}\right)$, $152.8\left(\mathrm{CH}^{\text {major }}\right), 154.0\left(\mathrm{CH}^{\text {minor }}\right), 218.9\left(\mathrm{C}^{\text {major }}+\mathrm{C}^{\text {minor }}\right)$; IR $v 3445(\mathrm{~m}), 3175(\mathrm{~m}), 2960(\mathrm{~m}), 2360(\mathrm{w}), 1730(\mathrm{~s}), 1595(\mathrm{~m})$, $1470(m), 1355(s), 1320(m), 1185(s), 1165(s), 1093(m), 815(s)$; Anal. Calcd. for $\mathrm{C}_{17} \mathrm{H}_{24} \mathrm{~N}_{2} \mathrm{O}_{3} \mathrm{~S}: \mathrm{C}, 60.7 ; \mathrm{H}, 7.2 ; \mathrm{N}, 8.3$; Found: C, 60.9; H, 7.2; N, 8.1. 


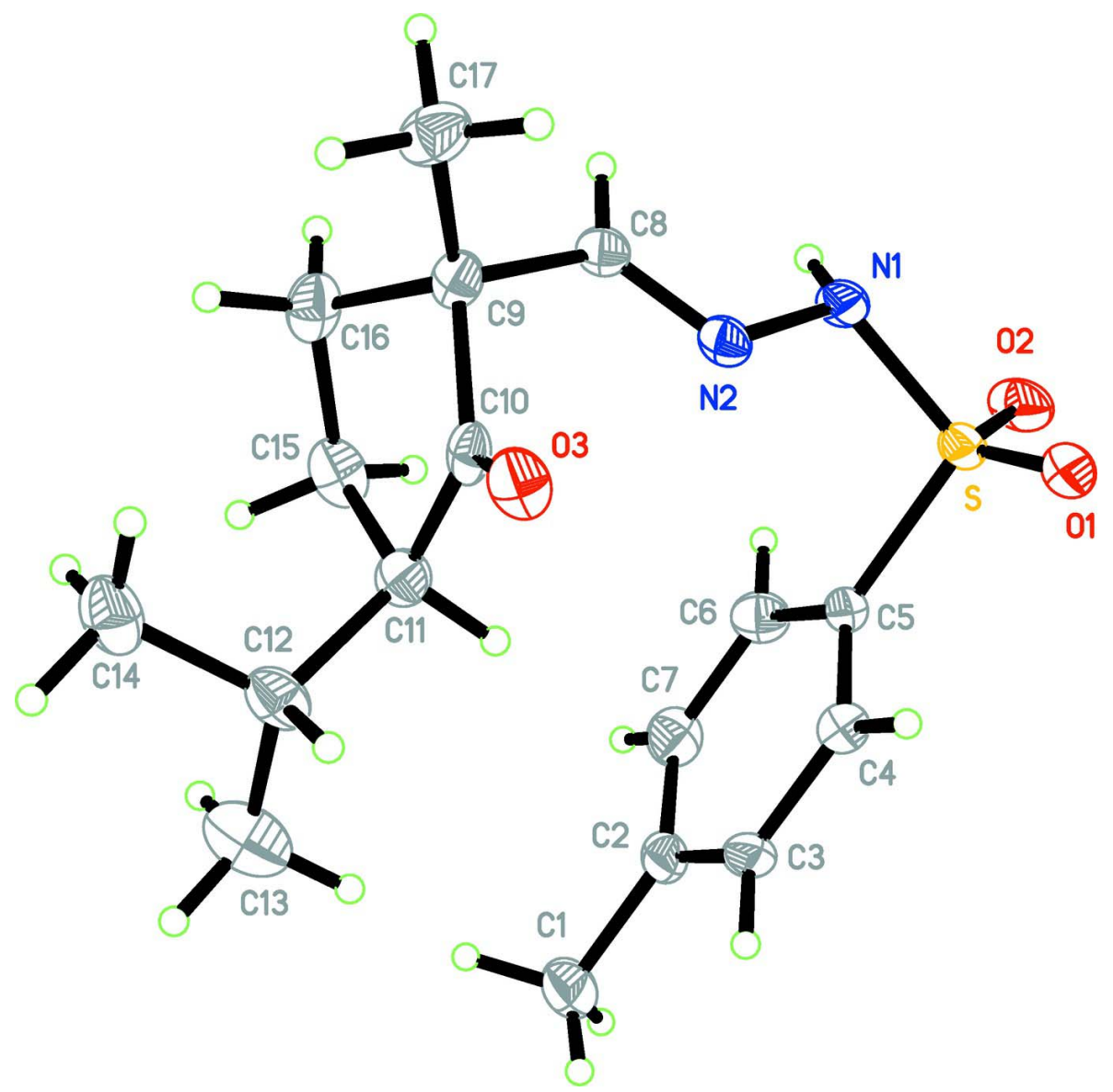

Figure 1

The molecular structure of the title compound, showing the labelling of all non-H atoms. Displacement ellipsoids are shown at the $30 \%$ probability level.

$(E)-N^{\prime}$-\{[(1R,3R)-3-Isopropyl-1-methyl-2-oxocyclopentyl]methylidene\}-4-methylbenzenesulfonohydrazide

\section{Crystal data}

$\mathrm{C}_{17} \mathrm{H}_{24} \mathrm{~N}_{2} \mathrm{O}_{3} \mathrm{~S}$

$M_{r}=336.44$

Monoclinic, $P 2_{1}$

$a=6.6198(8) \AA$

$b=16.8318(18) \AA$

$c=7.9506(9) \AA$

$\beta=97.141(11)^{\circ}$

$V=879.00(17) \AA^{3}$

Data collection

Oxford Diffraction Xcalibur Sapphire3 diffractometer

Radiation source: Enhance (Mo) X-ray Source Detector resolution: 16.0560 pixels $\mathrm{mm}^{-1}$ phi and $\omega$ scans

Absorption correction: multi-scan

(CrysAlis CCD; Oxford Diffraction, 2008)

$T_{\min }=0.98, T_{\max }=1.00$
$Z=2$

$F(000)=360$

$D_{\mathrm{x}}=1.271 \mathrm{Mg} \mathrm{m}^{-3}$

Mo $K \alpha$ radiation, $\lambda=0.71073 \AA$

$\mu=0.20 \mathrm{~mm}^{-1}$

$T=173 \mathrm{~K}$

Plate, colourless

$0.23 \times 0.10 \times 0.03 \mathrm{~mm}$

6620 measured reflections

3684 independent reflections

3185 reflections with $I>2 \sigma(I)$

$R_{\text {int }}=0.032$

$\theta_{\text {max }}=27.0^{\circ}, \theta_{\min }=2.4^{\circ}$

$h=-7 \rightarrow 8$

$k=-21 \rightarrow 21$

$l=-10 \rightarrow 9$ 


\section{Refinement}

Refinement on $F^{2}$

Least-squares matrix: full

$R\left[F^{2}>2 \sigma\left(F^{2}\right)\right]=0.044$

$w R\left(F^{2}\right)=0.091$

$S=1.01$

3684 reflections

216 parameters

1 restraint

Primary atom site location: structure-invariant direct methods

Secondary atom site location: difference Fourier map
Hydrogen site location: mixed

$\mathrm{H}$ atoms treated by a mixture of independent and constrained refinement

$w=1 /\left[\sigma^{2}\left(F_{\mathrm{o}}^{2}\right)+(0.0406 P)^{2}\right]$

where $P=\left(F_{\mathrm{o}}^{2}+2 F_{\mathrm{c}}{ }^{2}\right) / 3$

$(\Delta / \sigma)_{\max }<0.001$

$\Delta \rho_{\max }=0.21 \mathrm{e} \AA^{-3}$

$\Delta \rho_{\min }=-0.29$ e $\AA^{-3}$

Absolute structure: Flack $x$ determined using 1307 quotients $\left[\left(I^{+}\right)-\left(I^{-}\right)\right] /\left[\left(I^{+}\right)+\left(I^{-}\right)\right]$(Parsons \& Flack,2004)

Absolute structure parameter: $0.02(5)$

\section{Special details}

Experimental. Absorption correction: CrysAlis PRO, Agilent Technologies, Version 1.171.36.24 (release 03-12-2012 CrysAlis171. NET) (compiled Dec 3 2012,18:21:49) Empirical absorption correction using spherical harmonics, implemented in SCALE3 ABSPACK scaling algorithm.

Geometry. All e.s.d.'s (except the e.s.d. in the dihedral angle between two 1.s. planes) are estimated using the full covariance matrix. The cell e.s.d.'s are taken into account individually in the estimation of e.s.d.'s in distances, angles and torsion angles; correlations between e.s.d.'s in cell parameters are only used when they are defined by crystal symmetry. An approximate (isotropic) treatment of cell e.s.d.'s is used for estimating e.s.d.'s involving 1.s. planes.

Refinement. Refinement of $F^{2}$ against ALL reflections. The weighted $R$-factor $w R$ and goodness of fit $S$ are based on $F^{2}$, conventional $R$-factors $R$ are based on $F$, with $F$ set to zero for negative $F^{2}$. The threshold expression of $F^{2}>\sigma\left(F^{2}\right)$ is used only for calculating $R$-factors(gt) etc. and is not relevant to the choice of reflections for refinement. $R$-factors based on $F^{2}$ are statistically about twice as large as those based on $F$, and $R$ factors based on ALL data will be even larger.

Fractional atomic coordinates and isotropic or equivalent isotropic displacement parameters $\left(\AA^{2}\right)$

\begin{tabular}{lllll}
\hline & $x$ & $y$ & $z$ & $U_{\text {iso }} * / U_{\mathrm{eq}}$ \\
\hline $\mathrm{S}$ & $0.09218(13)$ & $0.45682(4)$ & $0.12604(10)$ & $0.0211(2)$ \\
$\mathrm{O} 1$ & $0.2056(4)$ & $0.41202(15)$ & $0.0179(3)$ & $0.0301(7)$ \\
$\mathrm{O} 2$ & $-0.1089(4)$ & $0.43231(14)$ & $0.1507(3)$ & $0.0328(7)$ \\
$\mathrm{O} 3$ & $0.7126(4)$ & $0.64917(14)$ & $0.0645(3)$ & $0.0263(6)$ \\
$\mathrm{N} 1$ & $0.0612(5)$ & $0.54744(16)$ & $0.0481(4)$ & $0.0201(7)$ \\
$\mathrm{N} 2$ & $0.2410(4)$ & $0.59285(16)$ & $0.0665(4)$ & $0.0190(6)$ \\
$\mathrm{C} 1$ & $0.6011(7)$ & $0.4966(3)$ & $0.8060(5)$ & $0.0363(10)$ \\
$\mathrm{H} 1 \mathrm{~A}$ & 0.6121 & 0.5531 & 0.8347 & $0.054^{*}$ \\
$\mathrm{H} 1 \mathrm{~B}$ & 0.7374 & 0.4746 & 0.8012 & $0.054^{*}$ \\
$\mathrm{H} 1 \mathrm{C}$ & 0.5358 & 0.4684 & 0.8926 & $0.054^{*}$ \\
$\mathrm{C} 2$ & $0.4754(6)$ & $0.4867(2)$ & $0.6364(5)$ & $0.0233(8)$ \\
$\mathrm{C} 4$ & $0.4451(5)$ & $0.4458(2)$ & $0.3415(4)$ & $0.0209(8)$ \\
$\mathrm{H} 4$ & 0.5048 & 0.4249 & 0.2483 & $0.025^{*}$ \\
$\mathrm{C} 3$ & $0.5595(5)$ & $0.4551(2)$ & $0.4991(4)$ & $0.0233(7)$ \\
$\mathrm{H} 3$ & 0.6986 & 0.4396 & 0.5133 & $0.028^{*}$ \\
$\mathrm{C} 5$ & $0.2427(5)$ & $0.46758(19)$ & $0.3241(4)$ & $0.0185(7)$ \\
$\mathrm{C} 6$ & $0.1547(6)$ & $0.4995(2)$ & $0.4585(5)$ & $0.0251(8)$ \\
$\mathrm{H} 6$ & 0.0157 & 0.5151 & 0.4435 & $0.030^{*}$
\end{tabular}




$\begin{array}{lllll}\text { C7 } & 0.2701(6) & 0.5084(2) & 0.6137(5) & 0.0277(9) \\ \text { H7 } & 0.2095 & 0.5295 & 0.7063 & 0.033^{*} \\ \text { C8 } & 0.2181(5) & 0.66745(19) & 0.0519(4) & 0.0190(7) \\ \text { H8 } & 0.0848 & 0.6889 & 0.0286 & 0.023^{*} \\ \text { C9 } & 0.3979(6) & 0.7225(2) & 0.0708(5) & 0.0206(8) \\ \text { C10 } & 0.5880(5) & 0.67705(19) & 0.1474(5) & 0.0199(8) \\ \text { C11 } & 0.6049(6) & 0.6771(2) & 0.3399(5) & 0.0229(8) \\ \text { H11 } & 0.5766 & 0.6223 & 0.3794 & 0.027^{*} \\ \text { C12 } & 0.8215(6) & 0.7019(2) & 0.4184(5) & 0.0260(9) \\ \text { H12 } & 0.9204 & 0.6689 & 0.3633 & 0.031^{*} \\ \text { C13 } & 0.8553(7) & 0.6835(3) & 0.6095(5) & 0.0462(12) \\ \text { H13A } & 0.9966 & 0.6954 & 0.6544 & 0.069^{*} \\ \text { H13B } & 0.8275 & 0.6271 & 0.6276 & 0.069^{*} \\ \text { H13C } & 0.7633 & 0.7161 & 0.6681 & 0.069^{*} \\ \text { C14 } & 0.8694(6) & 0.7886(2) & 0.3864(5) & 0.0331(10) \\ \text { H14A } & 0.7745 & 0.8227 & 0.4385 & 0.050^{*} \\ \text { H14B } & 0.8555 & 0.7986 & 0.2641 & 0.050^{*} \\ \text { H14C } & 1.0091 & 0.8005 & 0.4361 & 0.050^{*} \\ \text { C15 } & 0.4298(6) & 0.7325(2) & 0.3774(5) & 0.0283(9) \\ \text { H15A } & 0.3113 & 0.7012 & 0.4040 & 0.034^{*} \\ \text { H15B } & 0.4747 & 0.7677 & 0.4747 & 0.034^{*} \\ \text { C16 } & 0.3749(6) & 0.7811(2) & 0.2166(5) & 0.0282(9) \\ \text { H16A } & 0.4683 & 0.8268 & 0.2132 & 0.034^{*} \\ \text { H16B } & 0.2334 & 0.8011 & 0.2095 & 0.034^{*} \\ \text { C17 } & 0.4217(6) & 0.7612(2) & -0.0986(5) & 0.0296(10) \\ \text { H17A } & 0.4403 & 0.7200 & -0.1823 & 0.044^{*} \\ \text { H17B } & 0.5406 & 0.7964 & -0.0853 & 0.044^{*} \\ \text { H17C } & 0.2994 & 0.7923 & -0.1372 & 0.044^{*} \\ \text { H1N } & -0.051(6) & 0.572(2) & 0.079(5) & 0.027(11)^{*}\end{array}$

Atomic displacement parameters $\left(\AA^{2}\right)$

\begin{tabular}{lllllll}
\hline & $U^{11}$ & $U^{22}$ & $U^{33}$ & $U^{12}$ & $U^{13}$ & $U^{23}$ \\
\hline $\mathrm{S}$ & $0.0204(5)$ & $0.0192(4)$ & $0.0219(4)$ & $-0.0035(4)$ & $-0.0047(3)$ & $0.0012(4)$ \\
$\mathrm{O} 1$ & $0.0387(18)$ & $0.0257(13)$ & $0.0233(14)$ & $0.0052(12)$ & $-0.0065(13)$ & $-0.0048(11)$ \\
$\mathrm{O} 2$ & $0.0232(16)$ & $0.0337(15)$ & $0.0382(16)$ & $-0.0125(11)$ & $-0.0090(12)$ & $0.0108(12)$ \\
$\mathrm{O} 3$ & $0.0152(14)$ & $0.0305(14)$ & $0.0335(15)$ & $0.0027(11)$ & $0.0039(12)$ & $-0.0092(12)$ \\
$\mathrm{N} 1$ & $0.0120(16)$ & $0.0218(16)$ & $0.0252(17)$ & $-0.0016(12)$ & $-0.0024(13)$ & $0.0021(13)$ \\
$\mathrm{N} 2$ & $0.0129(16)$ & $0.0229(15)$ & $0.0209(16)$ & $-0.0022(12)$ & $0.0005(12)$ & $0.0003(12)$ \\
$\mathrm{C} 1$ & $0.046(3)$ & $0.032(2)$ & $0.027(2)$ & $-0.004(2)$ & $-0.009(2)$ & $-0.0023(18)$ \\
C2 & $0.029(2)$ & $0.0184(18)$ & $0.021(2)$ & $-0.0061(15)$ & $-0.0019(17)$ & $0.0027(14)$ \\
C4 & $0.0215(19)$ & $0.0194(19)$ & $0.0217(17)$ & $0.0011(16)$ & $0.0023(14)$ & $0.0007(15)$ \\
C3 & $0.0167(17)$ & $0.0217(16)$ & $0.0296(18)$ & $0.0004(17)$ & $-0.0053(14)$ & $0.0021(19)$ \\
C5 & $0.0200(18)$ & $0.0157(18)$ & $0.0188(16)$ & $-0.0015(14)$ & $-0.0018(14)$ & $0.0005(14)$ \\
C6 & $0.020(2)$ & $0.0289(19)$ & $0.026(2)$ & $0.0013(16)$ & $0.0034(17)$ & $0.0025(16)$ \\
C7 & $0.034(2)$ & $0.028(2)$ & $0.021(2)$ & $0.0013(17)$ & $0.0071(18)$ & $-0.0025(16)$ \\
C8 & $0.0119(18)$ & $0.0218(18)$ & $0.0229(18)$ & $0.0016(14)$ & $0.0008(14)$ & $-0.0003(15)$ \\
C9 & $0.0154(19)$ & $0.0183(18)$ & $0.029(2)$ & $-0.0004(14)$ & $0.0039(16)$ & $0.0001(15)$
\end{tabular}




\begin{tabular}{lllllll}
\hline & & & & & \\
$\mathrm{C} 10$ & $0.014(2)$ & $0.0154(17)$ & $0.031(2)$ & $-0.0055(14)$ & $0.0051(16)$ & $-0.0030(15)$ \\
$\mathrm{C} 11$ & $0.019(2)$ & $0.0229(19)$ & $0.027(2)$ & $-0.0016(15)$ & $0.0032(16)$ & $0.0008(16)$ \\
$\mathrm{C} 12$ & $0.017(2)$ & $0.035(2)$ & $0.026(2)$ & $0.0014(15)$ & $0.0024(17)$ & $-0.0061(17)$ \\
$\mathrm{C} 13$ & $0.037(3)$ & $0.067(3)$ & $0.034(3)$ & $-0.001(2)$ & $0.000(2)$ & $-0.002(2)$ \\
$\mathrm{C} 14$ & $0.021(2)$ & $0.038(2)$ & $0.041(2)$ & $-0.0060(17)$ & $0.0044(19)$ & $-0.0082(19)$ \\
$\mathrm{C} 15$ & $0.021(2)$ & $0.035(2)$ & $0.030(2)$ & $-0.0016(17)$ & $0.0063(17)$ & $-0.0097(18)$ \\
$\mathrm{C} 16$ & $0.020(2)$ & $0.0221(18)$ & $0.043(2)$ & $0.0017(15)$ & $0.0068(18)$ & $-0.0071(17)$ \\
$\mathrm{C} 17$ & $0.024(2)$ & $0.028(2)$ & $0.037(2)$ & $0.0000(16)$ & $0.0047(19)$ & $0.0094(17)$ \\
\hline
\end{tabular}

Geometric parameters $\left(\AA,{ }^{\circ}\right)$

\begin{tabular}{|c|c|c|c|}
\hline $\mathrm{S}-\mathrm{O} 1$ & $1.426(3)$ & $\mathrm{C} 9-\mathrm{C} 17$ & $1.522(5)$ \\
\hline $\mathrm{S}-\mathrm{O} 2$ & $1.431(3)$ & $\mathrm{C} 9-\mathrm{C} 10$ & $1.533(5)$ \\
\hline $\mathrm{S}-\mathrm{N} 1$ & $1.649(3)$ & $\mathrm{C} 9-\mathrm{C} 16$ & $1.544(5)$ \\
\hline $\mathrm{S}-\mathrm{C} 5$ & $1.765(3)$ & $\mathrm{C} 10-\mathrm{C} 11$ & $1.520(5)$ \\
\hline $\mathrm{O} 3-\mathrm{C} 10$ & $1.213(4)$ & $\mathrm{C} 11-\mathrm{C} 15$ & $1.546(5)$ \\
\hline $\mathrm{N} 1-\mathrm{N} 2$ & $1.407(4)$ & $\mathrm{C} 11-\mathrm{C} 12$ & $1.548(5)$ \\
\hline $\mathrm{N} 1-\mathrm{H} 1 \mathrm{~N}$ & $0.91(4)$ & $\mathrm{C} 11-\mathrm{H} 11$ & 1.0000 \\
\hline $\mathrm{N} 2-\mathrm{C} 8$ & $1.268(4)$ & $\mathrm{C} 12-\mathrm{C} 14$ & $1.522(6)$ \\
\hline $\mathrm{C} 1-\mathrm{C} 2$ & $1.503(5)$ & $\mathrm{C} 12-\mathrm{C} 13$ & $1.539(5)$ \\
\hline $\mathrm{C} 1-\mathrm{H} 1 \mathrm{~A}$ & 0.9800 & $\mathrm{C} 12-\mathrm{H} 12$ & 1.0000 \\
\hline $\mathrm{C} 1-\mathrm{H} 1 \mathrm{~B}$ & 0.9800 & $\mathrm{C} 13-\mathrm{H} 13 \mathrm{~A}$ & 0.9800 \\
\hline $\mathrm{C} 1-\mathrm{H} 1 \mathrm{C}$ & 0.9800 & C13-H13B & 0.9800 \\
\hline $\mathrm{C} 2-\mathrm{C} 3$ & $1.391(5)$ & $\mathrm{C} 13-\mathrm{H} 13 \mathrm{C}$ & 0.9800 \\
\hline $\mathrm{C} 2-\mathrm{C} 7$ & $1.398(5)$ & $\mathrm{C} 14-\mathrm{H} 14 \mathrm{~A}$ & 0.9800 \\
\hline $\mathrm{C} 4-\mathrm{C} 5$ & $1.380(5)$ & C14-H14B & 0.9800 \\
\hline $\mathrm{C} 4-\mathrm{C} 3$ & $1.390(5)$ & $\mathrm{C} 14-\mathrm{H} 14 \mathrm{C}$ & 0.9800 \\
\hline $\mathrm{C} 4-\mathrm{H} 4$ & 0.9500 & $\mathrm{C} 15-\mathrm{C} 16$ & $1.523(6)$ \\
\hline $\mathrm{C} 3-\mathrm{H} 3$ & 0.9500 & C15-H15A & 0.9900 \\
\hline $\mathrm{C} 5-\mathrm{C} 6$ & $1.387(5)$ & C15-H15B & 0.9900 \\
\hline $\mathrm{C} 6-\mathrm{C} 7$ & $1.376(5)$ & C16-H16A & 0.9900 \\
\hline C6- & 0.9500 & C16-H16B & 0.9900 \\
\hline $\mathrm{C} 7-\mathrm{H} 7$ & 0.9500 & C17-H17A & 0.9800 \\
\hline $\mathrm{C} 8-\mathrm{C} 9$ & $1.501(5)$ & C17-H17B & 0.9800 \\
\hline $\mathrm{C} 8-\mathrm{H} 8$ & 0.9500 & $\mathrm{C} 17-\mathrm{H} 17 \mathrm{C}$ & 0.9800 \\
\hline $\mathrm{O} 1-\mathrm{S}-\mathrm{O} 2$ & $119.95(16)$ & $\mathrm{O} 3-\mathrm{C} 10-\mathrm{C} 9$ & $123.9(3)$ \\
\hline $\mathrm{O} 1-\mathrm{S}-\mathrm{N} 1$ & $108.22(16)$ & $\mathrm{C} 11-\mathrm{C} 10-\mathrm{C} 9$ & 110.7 (3) \\
\hline $\mathrm{O} 2-\mathrm{S}-\mathrm{N} 1$ & $104.11(16)$ & $\mathrm{C} 10-\mathrm{C} 11-\mathrm{C} 15$ & $103.4(3)$ \\
\hline $\mathrm{O} 1-\mathrm{S}-\mathrm{C} 5$ & $108.13(16)$ & $\mathrm{C} 10-\mathrm{C} 11-\mathrm{C} 12$ & $110.8(3)$ \\
\hline $\mathrm{O} 2-\mathrm{S}-\mathrm{C} 5$ & $109.82(16)$ & $\mathrm{C} 15-\mathrm{C} 11-\mathrm{C} 12$ & $116.0(3)$ \\
\hline $\mathrm{N} 1-\mathrm{S}-\mathrm{C} 5$ & $105.67(15)$ & $\mathrm{C} 10-\mathrm{C} 11-\mathrm{H} 11$ & 108.8 \\
\hline $\mathrm{N} 2-\mathrm{N} 1-\mathrm{S}$ & $113.5(2)$ & $\mathrm{C} 15-\mathrm{C} 11-\mathrm{H} 11$ & 108.8 \\
\hline $\mathrm{N} 2-\mathrm{N} 1-\mathrm{H} 1 \mathrm{~N}$ & $116(2)$ & $\mathrm{C} 12-\mathrm{C} 11-\mathrm{H} 11$ & 108.8 \\
\hline $\mathrm{S}-\mathrm{N} 1-\mathrm{H} 1 \mathrm{~N}$ & $113(2)$ & $\mathrm{C} 14-\mathrm{C} 12-\mathrm{C} 13$ & $110.5(3)$ \\
\hline $\mathrm{C} 8-\mathrm{N} 2-\mathrm{N} 1$ & $116.0(3)$ & $\mathrm{C} 14-\mathrm{C} 12-\mathrm{C} 11$ & $113.1(3)$ \\
\hline $\mathrm{C} 2-\mathrm{C} 1-\mathrm{H} 1 \mathrm{~A}$ & 109.5 & $\mathrm{C} 13-\mathrm{C} 12-\mathrm{C} 11$ & $110.9(3)$ \\
\hline $\mathrm{C} 2-\mathrm{C} 1-\mathrm{H} 1 \mathrm{~B}$ & 109.5 & $\mathrm{C} 14-\mathrm{C} 12-\mathrm{H} 12$ & 107.3 \\
\hline
\end{tabular}




\begin{tabular}{|c|c|c|c|}
\hline $\mathrm{H} 1 \mathrm{~A}-\mathrm{C} 1-\mathrm{H} 1 \mathrm{~B}$ & 109.5 & $\mathrm{C} 13-\mathrm{C} 12-\mathrm{H} 12$ & 107.3 \\
\hline $\mathrm{C} 2-\mathrm{C} 1-\mathrm{H} 1 \mathrm{C}$ & 109.5 & $\mathrm{C} 11-\mathrm{C} 12-\mathrm{H} 12$ & 107.3 \\
\hline $\mathrm{H} 1 \mathrm{~A}-\mathrm{C} 1-\mathrm{H} 1 \mathrm{C}$ & 109.5 & $\mathrm{C} 12-\mathrm{C} 13-\mathrm{H} 13 \mathrm{~A}$ & 109.5 \\
\hline $\mathrm{H} 1 \mathrm{~B}-\mathrm{C} 1-\mathrm{H} 1 \mathrm{C}$ & 109.5 & $\mathrm{C} 12-\mathrm{C} 13-\mathrm{H} 13 \mathrm{~B}$ & 109.5 \\
\hline $\mathrm{C} 3-\mathrm{C} 2-\mathrm{C} 7$ & $118.4(3)$ & $\mathrm{H} 13 \mathrm{~A}-\mathrm{C} 13-\mathrm{H} 13 \mathrm{~B}$ & 109.5 \\
\hline $\mathrm{C} 3-\mathrm{C} 2-\mathrm{C} 1$ & $121.0(4)$ & $\mathrm{C} 12-\mathrm{C} 13-\mathrm{H} 13 \mathrm{C}$ & 109.5 \\
\hline $\mathrm{C} 7-\mathrm{C} 2-\mathrm{C} 1$ & $120.6(4)$ & $\mathrm{H} 13 \mathrm{~A}-\mathrm{C} 13-\mathrm{H} 13 \mathrm{C}$ & 109.5 \\
\hline $\mathrm{C} 5-\mathrm{C} 4-\mathrm{C} 3$ & $118.3(3)$ & $\mathrm{H} 13 \mathrm{~B}-\mathrm{C} 13-\mathrm{H} 13 \mathrm{C}$ & 109.5 \\
\hline $\mathrm{C} 5-\mathrm{C} 4-\mathrm{H} 4$ & 120.8 & $\mathrm{C} 12-\mathrm{C} 14-\mathrm{H} 14 \mathrm{~A}$ & 109.5 \\
\hline $\mathrm{C} 3-\mathrm{C} 4-\mathrm{H} 4$ & 120.8 & $\mathrm{C} 12-\mathrm{C} 14-\mathrm{H} 14 \mathrm{~B}$ & 109.5 \\
\hline $\mathrm{C} 4-\mathrm{C} 3-\mathrm{C} 2$ & $121.6(3)$ & $\mathrm{H} 14 \mathrm{~A}-\mathrm{C} 14-\mathrm{H} 14 \mathrm{~B}$ & 109.5 \\
\hline $\mathrm{C} 4-\mathrm{C} 3-\mathrm{H} 3$ & 119.2 & $\mathrm{C} 12-\mathrm{C} 14-\mathrm{H} 14 \mathrm{C}$ & 109.5 \\
\hline $\mathrm{C} 2-\mathrm{C} 3-\mathrm{H} 3$ & 119.2 & $\mathrm{H} 14 \mathrm{~A}-\mathrm{C} 14-\mathrm{H} 14 \mathrm{C}$ & 109.5 \\
\hline $\mathrm{C} 4-\mathrm{C} 5-\mathrm{C} 6$ & $121.4(3)$ & $\mathrm{H} 14 \mathrm{~B}-\mathrm{C} 14-\mathrm{H} 14 \mathrm{C}$ & 109.5 \\
\hline $\mathrm{C} 4-\mathrm{C} 5-\mathrm{S}$ & $119.7(3)$ & $\mathrm{C} 16-\mathrm{C} 15-\mathrm{C} 11$ & $105.5(3)$ \\
\hline $\mathrm{C} 6-\mathrm{C} 5-\mathrm{S}$ & $118.8(3)$ & $\mathrm{C} 16-\mathrm{C} 15-\mathrm{H} 15 \mathrm{~A}$ & 110.6 \\
\hline $\mathrm{C} 7-\mathrm{C} 6-\mathrm{C} 5$ & $119.5(3)$ & $\mathrm{C} 11-\mathrm{C} 15-\mathrm{H} 15 \mathrm{~A}$ & 110.6 \\
\hline $\mathrm{C} 7-\mathrm{C} 6-\mathrm{H} 6$ & 120.2 & $\mathrm{C} 16-\mathrm{C} 15-\mathrm{H} 15 \mathrm{~B}$ & 110.6 \\
\hline $\mathrm{C} 5-\mathrm{C} 6-\mathrm{H} 6$ & 120.2 & $\mathrm{C} 11-\mathrm{C} 15-\mathrm{H} 15 \mathrm{~B}$ & 110.6 \\
\hline $\mathrm{C} 6-\mathrm{C} 7-\mathrm{C} 2$ & $120.7(3)$ & $\mathrm{H} 15 \mathrm{~A}-\mathrm{C} 15-\mathrm{H} 15 \mathrm{~B}$ & 108.8 \\
\hline $\mathrm{C} 6-\mathrm{C} 7-\mathrm{H} 7$ & 119.7 & $\mathrm{C} 15-\mathrm{C} 16-\mathrm{C} 9$ & $104.6(3)$ \\
\hline $\mathrm{C} 2-\mathrm{C} 7-\mathrm{H} 7$ & 119.7 & $\mathrm{C} 15-\mathrm{C} 16-\mathrm{H} 16 \mathrm{~A}$ & 110.8 \\
\hline $\mathrm{N} 2-\mathrm{C} 8-\mathrm{C} 9$ & $121.2(3)$ & $\mathrm{C} 9-\mathrm{C} 16-\mathrm{H} 16 \mathrm{~A}$ & 110.8 \\
\hline $\mathrm{N} 2-\mathrm{C} 8-\mathrm{H} 8$ & 119.4 & $\mathrm{C} 15-\mathrm{C} 16-\mathrm{H} 16 \mathrm{~B}$ & 110.8 \\
\hline $\mathrm{C} 9-\mathrm{C} 8-\mathrm{H} 8$ & 119.4 & $\mathrm{C} 9-\mathrm{C} 16-\mathrm{H} 16 \mathrm{~B}$ & 110.8 \\
\hline $\mathrm{C} 8-\mathrm{C} 9-\mathrm{C} 17$ & $110.1(3)$ & $\mathrm{H} 16 \mathrm{~A}-\mathrm{C} 16-\mathrm{H} 16 \mathrm{~B}$ & 108.9 \\
\hline $\mathrm{C} 8-\mathrm{C} 9-\mathrm{C} 10$ & $109.5(3)$ & $\mathrm{C} 9-\mathrm{C} 17-\mathrm{H} 17 \mathrm{~A}$ & 109.5 \\
\hline $\mathrm{C} 17-\mathrm{C} 9-\mathrm{C} 10$ & $113.3(3)$ & C9- $\mathrm{C} 17-\mathrm{H} 17 \mathrm{~B}$ & 109.5 \\
\hline $\mathrm{C} 8-\mathrm{C} 9-\mathrm{C} 16$ & $108.5(3)$ & $\mathrm{H} 17 \mathrm{~A}-\mathrm{C} 17-\mathrm{H} 17 \mathrm{~B}$ & 109.5 \\
\hline $\mathrm{C} 17-\mathrm{C} 9-\mathrm{C} 16$ & $114.9(3)$ & $\mathrm{C} 9-\mathrm{C} 17-\mathrm{H} 17 \mathrm{C}$ & 109.5 \\
\hline $\mathrm{C} 10-\mathrm{C} 9-\mathrm{C} 16$ & $100.0(3)$ & $\mathrm{H} 17 \mathrm{~A}-\mathrm{C} 17-\mathrm{H} 17 \mathrm{C}$ & 109.5 \\
\hline $\mathrm{O} 3-\mathrm{C} 10-\mathrm{C} 11$ & $125.3(3)$ & $\mathrm{H} 17 \mathrm{~B}-\mathrm{C} 17-\mathrm{H} 17 \mathrm{C}$ & 109.5 \\
\hline $\mathrm{O} 1-\mathrm{S}-\mathrm{N} 1-\mathrm{N} 2$ & $-71.4(3)$ & $\mathrm{N} 2-\mathrm{C} 8-\mathrm{C} 9-\mathrm{C} 10$ & $-13.1(5)$ \\
\hline $\mathrm{O} 2-\mathrm{S}-\mathrm{N} 1-\mathrm{N} 2$ & $159.9(2)$ & $\mathrm{N} 2-\mathrm{C} 8-\mathrm{C} 9-\mathrm{C} 16$ & $-121.3(3)$ \\
\hline $\mathrm{C} 5-\mathrm{S}-\mathrm{N} 1-\mathrm{N} 2$ & $44.2(3)$ & $\mathrm{C} 8-\mathrm{C} 9-\mathrm{C} 10-\mathrm{O} 3$ & $97.8(4)$ \\
\hline $\mathrm{S}-\mathrm{N} 1-\mathrm{N} 2-\mathrm{C} 8$ & $-161.8(3)$ & $\mathrm{C} 17-\mathrm{C} 9-\mathrm{C} 10-\mathrm{O} 3$ & $-25.6(5)$ \\
\hline $\mathrm{C} 5-\mathrm{C} 4-\mathrm{C} 3-\mathrm{C} 2$ & $-0.9(5)$ & $\mathrm{C} 16-\mathrm{C} 9-\mathrm{C} 10-\mathrm{O} 3$ & $-148.4(3)$ \\
\hline $\mathrm{C} 7-\mathrm{C} 2-\mathrm{C} 3-\mathrm{C} 4$ & $0.7(5)$ & $\mathrm{C} 8-\mathrm{C} 9-\mathrm{C} 10-\mathrm{C} 11$ & $-86.2(4)$ \\
\hline $\mathrm{C} 1-\mathrm{C} 2-\mathrm{C} 3-\mathrm{C} 4$ & $-179.9(3)$ & $\mathrm{C} 17-\mathrm{C} 9-\mathrm{C} 10-\mathrm{C} 11$ & 150.5 \\
\hline $\mathrm{C} 3-\mathrm{C} 4-\mathrm{C} 5-\mathrm{C} 6$ & $1.2(5)$ & $\mathrm{C} 16-\mathrm{C} 9-\mathrm{C} 10-\mathrm{C} 11$ & $27.7(4)$ \\
\hline $\mathrm{C} 3-\mathrm{C} 4-\mathrm{C} 5-\mathrm{S}$ & $179.9(3)$ & $\mathrm{O} 3-\mathrm{C} 10-\mathrm{C} 11-\mathrm{C} 15$ & $169.9(3)$ \\
\hline $\mathrm{O} 1-\mathrm{S}-\mathrm{C} 5-\mathrm{C} 4$ & $10.2(3)$ & $\mathrm{C} 9-\mathrm{C} 10-\mathrm{C} 11-\mathrm{C} 15$ & $-6.1(4)$ \\
\hline $\mathrm{O} 2-\mathrm{S}-\mathrm{C} 5-\mathrm{C} 4$ & $142.7(3)$ & $\mathrm{O} 3-\mathrm{C} 10-\mathrm{C} 11-\mathrm{C} 12$ & $45.0(5)$ \\
\hline $\mathrm{N} 1-\mathrm{S}-\mathrm{C} 5-\mathrm{C} 4$ & $-105.5(3)$ & $\mathrm{C} 9-\mathrm{C} 10-\mathrm{C} 11-\mathrm{C} 12$ & $-131.0(3)$ \\
\hline $\mathrm{O} 1-\mathrm{S}-\mathrm{C} 5-\mathrm{C} 6$ & $-171.1(3)$ & $\mathrm{C} 10-\mathrm{C} 11-\mathrm{C} 12-\mathrm{C} 14$ & $68.4(4)$ \\
\hline $\mathrm{O} 2-\mathrm{S}-\mathrm{C} 5-\mathrm{C} 6$ & $-38.5(3)$ & $\mathrm{C} 15-\mathrm{C} 11-\mathrm{C} 12-\mathrm{C} 14$ & $-49.0(4)$ \\
\hline $\mathrm{N} 1-\mathrm{S}-\mathrm{C} 5-\mathrm{C} 6$ & $73.2(3)$ & $\mathrm{C} 10-\mathrm{C} 11-\mathrm{C} 12-\mathrm{C} 13$ & $-166.7(3)$ \\
\hline
\end{tabular}




$\begin{array}{llll}\mathrm{C} 4-\mathrm{C} 5-\mathrm{C} 6-\mathrm{C} 7 & -1.2(5) & \mathrm{C} 15-\mathrm{C} 11-\mathrm{C} 12-\mathrm{C} 13 & 75.8(4) \\ \mathrm{S}-\mathrm{C} 5-\mathrm{C} 6-\mathrm{C} 7 & -179.9(3) & \mathrm{C} 10-\mathrm{C} 11-\mathrm{C} 15-\mathrm{C} 16 & -18.9(4) \\ \mathrm{C} 5-\mathrm{C} 6-\mathrm{C} 7-\mathrm{C} 2 & 0.9(6) & \mathrm{C} 12-\mathrm{C} 11-\mathrm{C} 15-\mathrm{C} 16 & 102.6(4) \\ \mathrm{C} 3-\mathrm{C} 2-\mathrm{C} 7-\mathrm{C} 6 & -0.7(5) & \mathrm{C} 11-\mathrm{C} 15-\mathrm{C} 16-\mathrm{C} 9 & 37.0(4) \\ \mathrm{C} 1-\mathrm{C} 2-\mathrm{C} 7-\mathrm{C} 6 & 179.9(3) & \mathrm{C} 8-\mathrm{C} 9-\mathrm{C} 16-\mathrm{C} 15 & 75.9(4) \\ \mathrm{N} 1-\mathrm{N} 2-\mathrm{C} 8-\mathrm{C} 9 & 179.3(3) & \mathrm{C} 17-\mathrm{C} 9-\mathrm{C} 16-\mathrm{C} 15 & -160.4(3) \\ \mathrm{N} 2-\mathrm{C} 8-\mathrm{C} 9-\mathrm{C} 17 & 112.1(4) & \mathrm{C} 10-\mathrm{C} 9-\mathrm{C} 16-\mathrm{C} 15 & -38.7(3)\end{array}$

Hydrogen-bond geometry $\left(A,{ }^{\circ}\right)$

\begin{tabular}{lllll}
\hline$D-\mathrm{H} \cdots A$ & $D-\mathrm{H}$ & $\mathrm{H} \cdots A$ & $D \cdots A$ & $D-\mathrm{H} \cdots A$ \\
\hline $\mathrm{N} 1-\mathrm{H} 1 N \cdots \mathrm{O}^{\mathrm{i}}$ & $0.91(4)$ & $2.03(4)$ & $2.889(4)$ & $158(3)$ \\
\hline
\end{tabular}

Symmetry code: (i) $x-1, y, z$. 